\title{
ESTUDO EXPLORATÓRIO SOBRE GERENCIAMENTO DE PROJETOS EM STARTUPS PERNAMBUCANAS
}

\author{
Karla Thais Chaves da Silva - karlakt25@gmail.com \\ Ivaldir de Farias Junior - ivaldirjr@gmail.com² \\ Hermano Moura - hermano@cin.ufpe.br ${ }^{3}$
}

\begin{abstract}
Resumo - As práticas de engenharia de software são atividades desafiadoras no âmbito das startups, dada a sua origem limitada e necessidade de criar produtos inovadores e de alta tecnologia. Neste cenário, destaca-se a importância da gestão de projetos, pois estes são iniciados para criar mudanças, desenvolver novos produtos, estabelecer novos processos, ou ainda criar uma nova organização. O objetivo deste trabalho é identificar as técnicas, práticas e ferramentas utilizadas no gerenciamento de projetos em startups pernambucanas com o propósito de propiciar a essas organizações informações pertinentes para que as mesmas possam desenvolver-se em meio às incertezas da indústria. O método de pesquisa é formado por uma pesquisa exploratória e um survey. No survey, foram obtidas respostas de 13 startups pernambucanas, possibilitando demonstrar o perfil dessas organizações, dos respondentes e do desenvolvimento de software.
\end{abstract}

Palavras-chave: Gerenciamento de Projetos, Startups, Práticas.

\section{Exploratory study on project management in pernambucan Startup}

\begin{abstract}
Software engineering practices are challenging activities within startups, given their limited origin and the need to create innovative and high-tech products. In this case it is notorious the importance of project management which aims to create changes, develop new products, establish new processes or even create a new organization. The aim of this paper is to identify the techniques, practices and tools used in project management in Pernambuco startups with the purpose of providing these organizations with a set of pertinent tools so that they can be developed amid the uncertainties of the industry. The research method used in this work is formed by an exploratory research and a survey. Through the survey, responses were obtained from 13 startups from Pernambuco, making it possible to demonstrate the profile of these organizations.
\end{abstract}

Keywords: Project Management, Startups, Practices.

Data de Aceitação: 30/05/2020 


\section{Introdução}

Startups são consideradas empresas nascentes de base tecnológica, que possuem na inovação tecnológica disruptiva os fundamentos de sua estratégia competitiva. Entre as principais características de tais negócios estão o caráter de organização temporária com potencial de rápido crescimento, os quais atuam em um ambiente de extrema incerteza, em busca de um modelo de negócios que possa tornar-se repetível e escalável (ASSOCIAÇÃO BRASILEIRA DE STARTUPS, 20I4; BLANK, 20I3).

Cerca de Io mil startups em 2012 somaram cerca de $\mathrm{R} \$ 2$ bilhões, o que representa 0,4\% do PIB brasileiro (DOM CABRAL, 20I5). Porém estas empresas têm complicações para adaptarem-se às mudanças que, são constantes, ocorrem em alta velocidade e em intensa demanda. Estima-se que a mortalidade dessas organizações é alta, cerca de 50\% deles não sobrevivem aos primeiros quatro anos de operação (DOM CABRAL, 2015).

Muitos destes projetos fracassam por diversas razões, como falta de recursos, erros na estimativa de tempo, desconhecimento sobre negócios, clientes sem a visão do que desejam, escopos mal elaborados, prazos irreais, gerentes de projetos despreparados entre outros motivos.

Neste cenário, Shenhar et al., (2002), ressaltam a importância da gestão de projetos. Estes são iniciados para criar mudanças, desenvolver novos produtos, estabelecer novos processos, ou ainda criar uma nova organização. Sem projetos, as organizações se tornariam obsoletas, e incapazes de lidar com o atual ambiente competitivo (SHENHAR et al., 2002) .Projetos conduzidos em ambientes de incerteza representam uma questão chave em gerenciamento de projetos que ainda não foi resolvida e apresentam os desafios de fazer planejamento para resultados incertos, equilibrar flexibilidade com confiabilidade e responsabilidade, balancear qualidade de decisão contra velocidade de decisão e congelamento de escopo durante as rápidas mudanças (Larson e Gray, 20II). Adicionalmente, em ambientes dinâmicos, normalmente os projetos estão interligados entre si, e as altas taxas de mudança, fazem o planejamento ser cada vez mais desafiador (Collyer e Warren, 2009).

Entretanto, as startups nacionais estão atraindo mais atenção a cada ano e, com a ajuda de investidores, aceleradoras e incubadoras, o cenário vem se expandindo aos novos empreendedores.Neste cenário, Pernambuco é um importante polo de empreendedorismo brasileiro, destacando-se por startups de relevância nacional, além de estar presente em diversas cidades do estado, como: a capital Recife, Caruaru, Petrolina, Garanhuns (ABStartups, 20I8). Segundo a Radiografia do Ecossistema de Startups Brasileiras realizada pela ABStartups (20I8), o estado lidera a criação de negócios desse tipo na região nordeste além de possuir um importante comunidade de startups que apoia práticas colaborativas e aprendizagem de tecnologia, design e empreendedorismo em todo o ecossistema de inicialização.

Assim sendo, se faz necessário que as empresas inseridas em ambientes com alto grau de insegurança possam identificar seus efeitos e causas e ainda utilizar resoluções precisas desses problemas fundamentais para as tomadas de decisões apropriadas à situação das instituições.

Ao ressaltar na pesquisa a busca por técnicas, práticas e ferramentas utilizadas no desenvolvimento de software em startups, este estudo tem por objetivo criar um alicerce de conhecimento que pode ser transferido para a indústria de forma ágil, podendo, no curto prazo, 
fornecer suporte para startups que estejam iniciando no mercado ou, até mesmo, em fase de crescimento. Ao investigar quais as técnicas, práticas e ferramentas são as mais utilizadas pela indústria, estas organizações poderiam tomar decisões melhores embasadas empiricamente validadas, orientando assim as suas escolhas.

\section{Referencial Teórico}

\subsection{Gestão de projetos}

Foi na segunda metade do século XX que surgiram as principais associações que visam organizar os conhecimentos e interessados em gerenciamento de projetos. A mais conhecida delas, que formalizou a prática de gerenciamento de projetos e criou a mais relevante certificação na área, chama-se Project Management Institute, ou apenas PMI, que é atualmente a maior associação não governamental dedicada ao gerenciamento de projetos com mais de 700.000 membros no mundo (Pmi, 20I6). Em menor escala, mas com objetivo similar de formular e codificar as práticas de gerenciamento de projetos existe também o International Project Management Association, ou IPMA, que já atua em mais de 50 países (Ipma, 20I6).

Convencionalmente, a definição de projeto é uma iniciativa única com começo e fim pré-determinados, conduzido por pessoas para atingir os objetivos estabelecidos segundo parâmetros de custo, cronograma e qualidade (Buchanan e Boddy, 1992). O gerenciamento de projetos, por sua vez, fundamenta-se no planejamento, programação e controle de uma série de tarefas integradas para atingir seus objetivos com êxito, em prol dos participantes da iniciativa (Kerzner, 2006).

O gerenciamento de projetos diferencia-se do gerenciamento de operações, uma vez que os projetos são pontuais, possuem data para início e fim, trazem melhoria relevante (diferente de simples evolução), criam "desequilíbrio", utilizam times que estarão temporariamente alocados para aquela iniciativa, "começam com poucos precedentes", são orientados para o objetivo e trazem componentes de risco (Williams, 2005)

\subsection{Desenvolvimento de startups de software}

Uma startup consiste em um grupo de pessoas buscando um desenvolvimento de software em startups modelo de negócios repetível e escalável, e que não necessariamente tem certeza a respeito desse modelo de negócios (GITAHY, 20IO).

Crowne (2002) caracteriza o ciclo de vida de uma startup por meio de uma evolução em três fases: inicialização, estabilização e crescimento. Crowne (2002) determina a fase de inicialização como a fase entre a concepção do produto até a realização da primeira venda. Nesta fase, o empreendedor precisa identificar uma oportunidade de mercado e então, por meio da tecnologia disponível, determinar uma maneira de aproveitá-la (Giardino et al., 20I4; Nguyen-Duc et al., 20I6). A fase de estabilização começa no momento em que o primeiro consumidor recebe o produto, durando até o momento em que o produto está estável o suficiente para ser vendido a um novo consumidor sem causar sobrecarga no desenvolvimento do produto (Nguyen-Duc et al., 20I6). É nesta fase que o empreendedor, geralmente, precisa buscar por investidores externos para financiar suas operações. Então, a fase de crescimento se inicia exatamente ao fim da fase de estabilização e acaba quando as taxas de crescimento e fatia de mercado estão estáveis e 
todos os processos necessários para o desenvolvimento e venda do produto estão estabilizados (Klepper, 1996). Ao fim destas fases a startup se torna uma organização madura.

Startups são flexíveis por natureza e, devido a isto, relutantes em adotar processos e medidas burocráticas (Sutton, 2000). De acordo com Giardino et al.,20I4, devido ao dinamismo do ambiente das startups, as metodologias ágeis são o tipo de processo mais viável, com destaque para as metodologias Lean/Lean Startup, Scrum, Extreme Programming (XP), Experiment Driven Development (EDD), Feature Driven Development (FDD) e Scrumban. Alinhada às metodologias ágeis se encontra a metodologia lean, que prega uma aprendizagem rápida ao identificar as principais partes do negócio e desenvolver um MVP a partir disto, permitindo que seja possível testar e modificar rapidamente o produto de acordo com o feedback recebido (Cavalcante, 20I8).

Outro aspecto relevante para a manutenção e o desenvolvimento das startups de software é a existência de ecossistemas, tema este que vem ganhando atenção e sendo estudado recentemente (Santos, 20I6). Em seu trabalho Santos (20I6) aponta que os ecossistemas criam um ambiente favorável a prosperidade destas organizações, dado que permite uma maior interação entre estas startups, permitindo que se organizem de forma conjunta. Além do mais, o agrupamento destas organizações atrai a atenção de mão de obra qualificada e de potenciais investidores.

\subsection{Startups e gerenciamento de projetos}

É comum as organizações utilizarem projetos como forma de investigar novas tecnologias e mercados (Von Hippel, 1977), isso acontece porque elas precisam superar a incerteza daquilo que ainda não foi explorado e precisam identificar ou criar oportunidades (Frederiksen e Davies, 2008). As empresas desenvolvem suas capacidades através de flexibilidade e adaptabilidade rápida requerida por esses projetos (Ethiraj et al., 2005).

Cada vez há mais estudos para mostrar como as empresas estão usando os projetos para melhorar seu desempenho e também para experimentar a inovação em seus negócios (Frederiksen e Davies, 2008). Por serem temporários e flexíveis, os projetos permitem a exploração de recursos e capacidades já disponíveis para buscar vantagem competitiva. Frederiksen e Davies (2008) classificam isso de gestão administrativa de projetos que seja adaptável e responsiva ao ambiente que está inserida. Eles também permitem explorar novas formas de desenvolver a competitividade, se aventurando por novos mercados ao fazer uso da tecnologia como forma de buscar, descobrir e testar diferentes oportunidades.

\section{Metodologia}

No intuito de identificar as técnicas, práticas e ferramentas utilizadas no gerenciamento de projetos das startups do estado de Pernambuco, desenvolveu-se um estudo de natureza exploratória, com abordagem quantitativa. $\mathrm{O}$ trabalho foi desenvolvido em duas etapas: na primeira fase foi realizado um estudo exploratório através da Revisão da Literatura e na segunda fase foi realizado um Estudo empírico operacionalizado através de um Survey.

Neste trabalho, a fundamentação teórica teve como objetivo possibilitar a visualização do estado da arte e então suportar o desenvolvimento das demais etapas, além de gerar subsídio 
para a construção do Survey. Nesta etapa, foram tratadas as principais áreas envolvidas na pesquisa, sendo elas: Startups e Gestão de desenvolvimento de Software em Startups.

Neste estudo, o survey será guiado com o objetivo de avaliar perspectivas, baseado nas informações coletadas por meio da pesquisa exploratória. Portanto, o survey pode ser classificado como exploratório, porque intenta analisar uma área de pesquisa pouco explorada (Gil, 1999). Em relação ao modo de coleta de dados, caracteriza-se como corte transversal, pois a coleta ocorrerá em apenas um dado momento (Sampieri et al., 20I0). A população estudada para a aplicação do survey serão quaisquer startups que possuam o desenvolvimento de software como atividade principal ou como uma de suas atividades chave e que estejam localizadas na área geográfica delimitada.

\subsection{Condução da pesquisa exploratória}

Em relação a técnica utilizada para a coleta dos dados, a mesma é caracterizada como de corte transversal, uma vez que a compilação dos dados ocorreu em um período específico de tempo (Sampieri et al., 20I0). O survey foi conduzido de acordo com o método proposto por Forza (2002), que é subdivido em quatro etapas: Planejamento, Teste do experimento de Coleta, Coleta dos Dados e Análise dos Resultados.

\subsubsection{Planejamento}

A fase de Planejamento busca a prevenção de problemas durante as fases posteriores do survey e busca garantir a qualidade do processo de pesquisa. Esta etapa é composta das seguintes atividades: seleção de contexto, seleção dos participantes e desenvolvimento dos instrumentos de pesquisa (Cavalcante, 20I8).

\subsubsection{Seleção da Amostra}

Para a seleção da Amostra foi considerada qualquer startup de Pernambuco que tenham entre as suas atividades principais o desenvolvimento de software. Não foi definida nenhuma restrição em relação ao domínio de atuação da startup. A listagem de startups foi obtida por meio de sites de associações e agrupamentos, como por exemplo a Associação Brasileira de Startups(20I8) e Liga Insights (20I9). Todas as startups encontradas, considerando o contexto definido, foram convidadas via e-mail e redes sociais a participarem da pesquisa.

\subsubsection{Desenvolvimento dos Instrumentos de Pesquisa}

O instrumento de pesquisa consiste no Questionário (Survey). O Questionário indica as questões que compõem o survey, desenvolvidas de acordo o propósito da pesquisa, ele está estruturado em 4 seções:

i. descrição do respondente, buscando identificar o perfil do respondente do questionário;

ii. descrição da startup, que visa identificar o perfil da organização e em qual estágio de vida se encontra;

iii. desenvolvimento de software, que tem por intuito reconhecer os métodos, práticas, técnicas e ferramentas utilizadas durante as atividades de gerenciamento dentro da 
startup.

\subsubsection{Teste do instrumento de coleta}

O Teste do instrumento de coleta pretende verificar a execução do que foi planejado. Desta forma, a mostragem foi conduzida com a meta de analisar o comportamento do instrumento de pesquisa em uma situação real de coleta de dados, proporcionando averiguar se as questões estavam compreensíveis, se as opções de respostas estavam completas e se o mesmo estava suficientemente sucinto, de forma a facilitar que fosse respondido e, assim, aumentar a adesão.

\subsubsection{Coleta de Dados}

A Coleta de Dados tem como finalidade agrupar as informações relevantes ao survey, proporcionando as informações necessárias para o cumprimento do objetivo da pesquisa.

A Coleta de Dados foi realizada utilizando a ferramenta de formulários do Google (forms.google.com). A escolha de uma ferramenta on-line para a coleta dos dados possibilita uma maior abrangência e facilita a sua divulgação, dado que startups que responderam o questionário podem encaminhar o link de acesso do mesmo para outras startups que ainda não participaram da pesquisa.

\subsubsection{Análise dos Resultados}

A Análise dos Resultados é essencial para os objetivos da pesquisa, pois possibilita investigar os dados por diversos ângulos e também compará-los, de forma a produzir novos conhecimentos. Neste survey, os dados foram estruturados e entrelaçados conforme as visões desejadas, permitindo destacar os fatos mais importantes e possibilitando o compreendimento das informações coletadas.

\subsection{Demografia da Pesquisa}

Com a utilização da ferramenta de edição de planilhas Excel foi possível perceber a existência de dados inconsistentes (outliers). Após a exclusão dos outliers, sendo I por duplicidade, o total da amostra foi reduzido para I3 participantes. O questionário foi enviado para I38 startups diferentes, pois das I8I empresas existentes em Pernambuco, 30 foram excluídas por não executarem atividades de desenvolvimento de software. Das I5I organizações restantes foram excluídas um total I3 devido à falta de canais de comunicação com as mesmas. Desta forma, o total de participantes da pesquisa foi I4 startups e a taxa de resposta da pesquisa foi de $9,42 \%$.

\section{Resultados}

Neste capítulo são expostos as etapas e os resultados do estudo empírico conduzido no trabalho, sendo este um survey exploratório. A pesquisa foi realizada com startups pernambucanas de desenvolvimento de software, sendo observadas as suas características, as características do indivíduo que respondeu o questionário e aspectos técnicos relacionados ao gerenciamento de projetos dentro das empresas. 


\subsection{Caracterização de Startup}

Essa seção visa caracterizar o perfil das startups que responderam o questionário, onde são exibidos o ano de fundação da startup, qual o seu nível de maturidade, se a startup faz parte de algum grupo ou rede de colaboração (ecossistema, incubadora, arranjos produtivos, etc.), se a startup possui algum tipo de investidor externo e quantos colaboradores a startup possui.

O gráfico I apresenta a distribuição das startups de acordo com o seu respectivo ano de fundação. $\mathrm{O}$ ano de 2018 é o que apresenta o maior número de startups fundadas, com 4, seguido de 20I6, com 3. Em seguida tem -se 20I2 com 2. Os anos de 20IO, 20II, 2015 e 2017 aparecem com startup fundada cada.

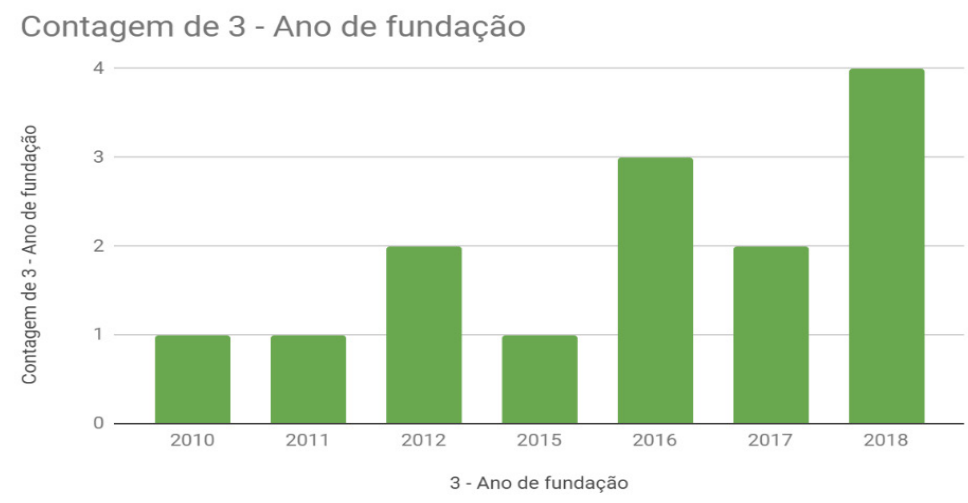

Gráfico I - Ano de fundação das startups

A Gráfico 2 apresenta o número de colaboradores das startups. Como forma de classificação, as faixas de número de colaboradores por startup foram definidas de acordo com os portes utilizados pelo Serviço Brasileiro de Apoio às Micro e Pequenas Empresas (SEBRAE), onde empresas até 9 colaboradores são considerados como microempresas, de Io até 49 colaboradores são consideradas de pequeno porte, de 50 a 99 colaboradores são consideradas de porte médio e de IOO ou mais colaboradores são consideradas como grande.

Deste modo, o Gráfico 2 demonstra que $78,4 \%$ respondentes são enquadradas como microempresas, enquanto 2I,6 \% são caracterizadas como empresas de grande porte. Assim podemos verificar que a grande maioria das startups participantes é formada por microempresas, ou seja, possuem no máximo 9 colaboradores. 


\section{4 - Quantidade de funcionários \\ 14 respostas}

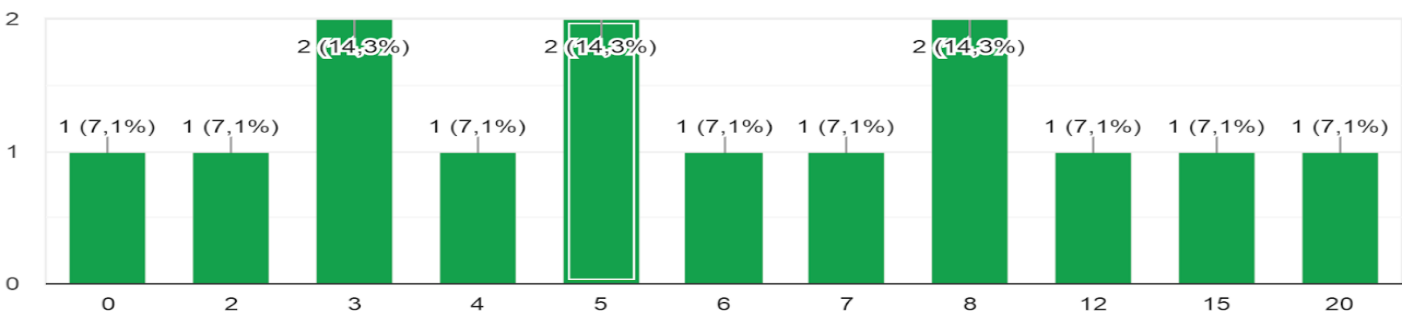

Gráfico 2 -Número de colaboradores das startups

Em relação ao nível de maturidade, das startups participantes. Conforme ilustra o gráfico 3, é possível perceber que a maioria das empresas em fase de crescimento.

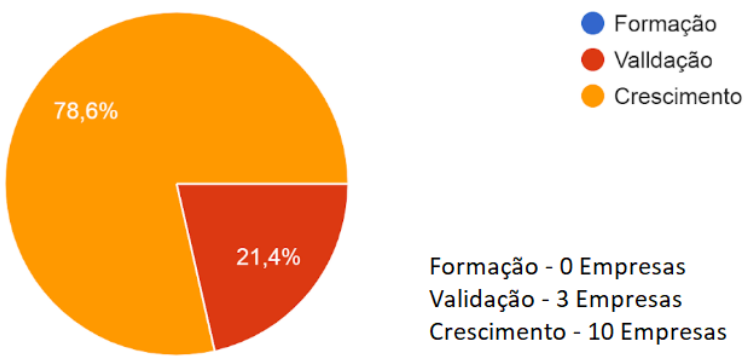

Gráfico 3 - Nível de maturidade das empresas

A tabela or apresenta uma relação entre o ano de fundação das startups e o seu nível de maturidade, exibindo pelo ano de fundação o percentual de startups que se encontra em cada nível de maturidade. A tabela permite verificar que todas as startups fundadas em 20II e 2012 estão em fase de crescimento, ou seja, estão mais próximas de se tornar empresas maduras.

Tabela I- Relação entre de fundação e o nível de maturidade

\begin{tabular}{|c|c|c|c|}
\hline Ano & Em fase de início & Em fase de estabilização & Em fase de crescimento \\
\hline 2011 & - & - & $10 \%$ \\
\hline 2012 & - & - & $20 \%$ \\
\hline 2013 & - & - & - \\
\hline 2014 & - & - & - \\
\hline 2015 & - & - & $10 \%$ \\
\hline 2016 & - & $33,3 \%$ & $20 \%$ \\
\hline 2017 & - & $33,3 \%$ & $10 \%$ \\
\hline 2018 & - & $33,3 \%$ & $30 \%$ \\
\hline
\end{tabular}


Ao serem questionadas se fazem parte de algum grupo ou rede de colaboração (ecossistema, incubadora, arranjos produtivos, etc), 57,1\% das organizações responderam que sim, enquanto $42,9 \%$ responderam que não fazem parte. Das empresas que indicaram resposta afirmativa, metade informaram colaboração do Porto Digital, tanto em Recife quanto a unidade do agreste do estado, o Armazém da Criatividade. Em outra questão, em relação a startup possuir ou não algum tipo de investidor externo, 50 \% indicaram que sim, enquanto as que não possuem constam com $50 \%$.

\section{Caracterização dos respondentes}

Essa seção apresenta o tempo de experiência com desenvolvimento de software e o grau de formação do respondente. Esses elementos visam caracterizar o perfil do respondente do questionário.

O gráfico 4 apresenta o tempo de experiência dos respondentes com desenvolvimento de software. É possível verificar que $78,6 \%$ possuem mais do que 5 anos de experiência, indicando que a grande maioria dos envolvidos nas startups possuem uma quantidade considerável de experiência com desenvolvimento de software. Do restante, 7,I\% possuem entre 3 e 5 anos de experiência e I4,3\% possuem entre I e 3 anos.

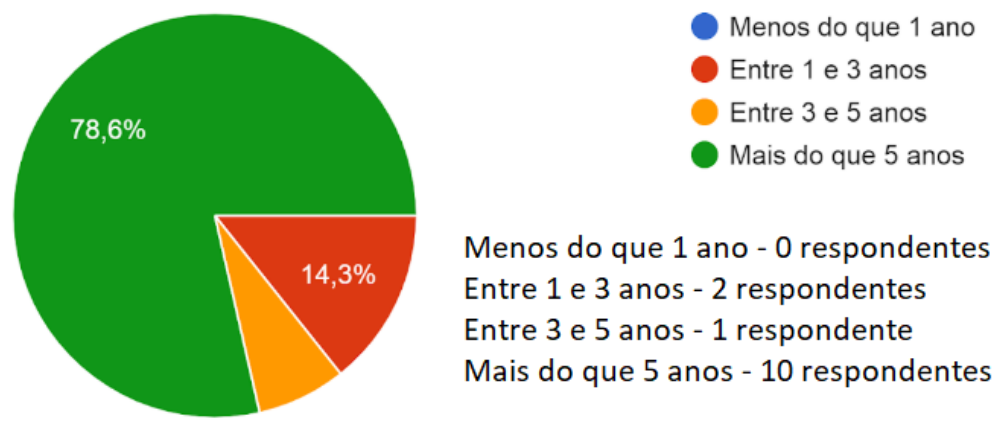

Gráfico 4 -Tempo de experiência dos respondentes com desenvolvimento de software

No que se refere ao grau de formação, nenhum dos respondentes indicou Curso Técnico,Doutorando ou Doutor. sendo que 7,I\% é Graduando, 28,6\% é Graduado, 42,9\% é Especialista, I4,3\% Mestrando, 7,I \% Mestre, conforme ilustrado pelo Gráfico 5. Isto comprova que boa parte das pessoas que estão envolvidas com startups são recém-formadas ou acabaram de terminar uma pós-graduação, ou então são pessoas que decidiram não investir em uma carreira acadêmica além da graduação e da especialização. 


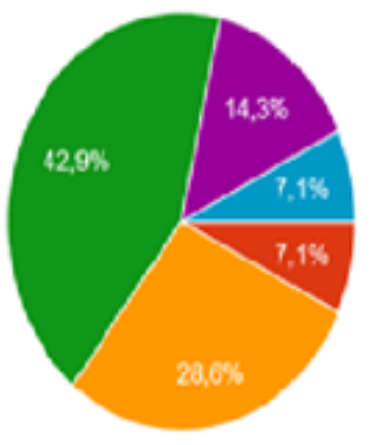

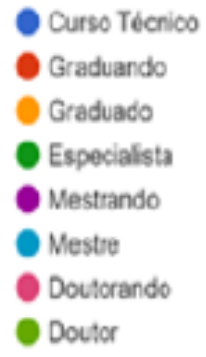

Curso Técnico - 0 respondentes

Graduando -1 respondente

Graduado -4 respondentes

Especialista -6 respondentes

Mestrando - 1 respondente

Mestre -1 respondente

Doutorando - 0 respondentes

Doutor - 0 respondentes

Gráfico 5 - Grau de formação do respondente

\subsection{Desenvolvimento de Software em Startups}

Essa seção apresenta se a startup utiliza metodologias ágeis e/ou metodologias tradicionais e quais, apresenta também quais as práticas e técnicas são utilizadas, quais tipos de testes, quais ferramentas de controle de versão e configuração, quais ferramentas de gerenciamento de projeto, quais suítes/plataformas de desenvolvimento e quais ferramentas de gestão do conhecimento as startups utilizam. Esses elementos visam caracterizar as atividades de desenvolvimento de software das startups que responderam o questionário.

A totalidade das empresas informaram utilizar metodologias ágeis. O Gráfico 6 apresenta as metodologias ágeis utilizadas pelas startups. A metodologia Scrum foi indicada por $85,7 \%$ startups, seguida pela metodologia Lean/Lean Startup que foi indicada por $28,6 \%$, a seguir aparece a metodologia Test Driven Development (TDD) com 2I,4\% das indicações, Extreme Programming (XP) com I4,3\% indicações, a metodologia Experiment Driven Development (EDD) e Kanban ambas com das 7,I\% indicações. Feature Driven Development (FDD) não obteve indicações.

Para efeitos da pesquisa o Kanban é considerado como uma técnica, e não como uma metodologia, inclusive sendo uma das opções em outra questão do questionário. Entretanto, não é incomum que a técnica seja encarada como uma metodologia por diversas organizações.

13- Se sim, qual(is)?

14 respostas

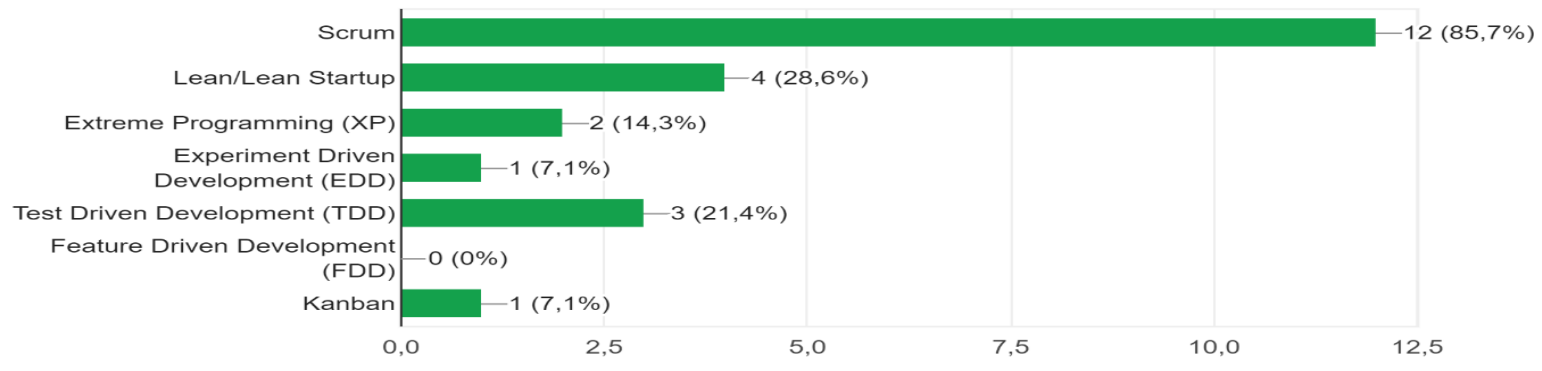

Gráfico 6 - Metodologias ágeis utilizadas pelas startups 
Quanto ao uso de métodos tradicionais, os resultados demonstram que, das startups participantes, apenas 2I,4\% utilizam métodos tradicionais, enquanto 78,6\% não utilizam. Em conjunto com os dados a respeito do uso de métodos ágeis, os dados de uso de métodos tradicionais demonstram que a maioria das startups de Pernambuco utiliza apenas métodos ágeis para desenvolvimento de software. No gráfico 7 são apresentadas quais metodologias tradicionais são utilizadas. No contexto do survey a resposta não é restrita, dando ao respondente a opção de escolher mais de uma alternativa proposta, oque significa a utilização de mais de uma metodologia. O modelo cascata não possui indicações, PDCA aparece com 50\% das indicações enquanto Rational Unified Process e PMBOK ilustram 25\% das indicações.
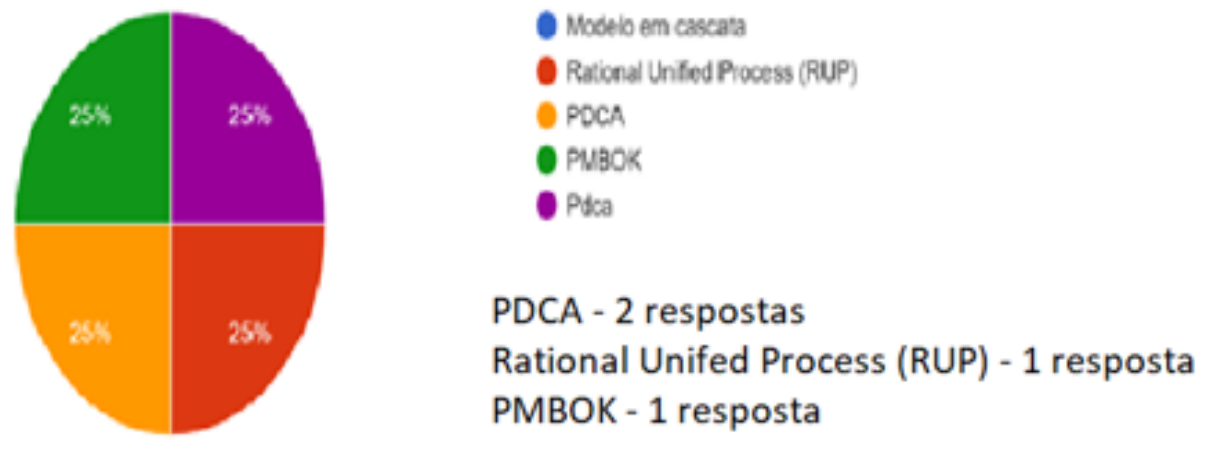

Gráfico 7 - Metodologias tradicionais utilizadas por startups

O Gráfico 8 apresenta quais são as práticas utilizadas pelas startups participantes da pesquisa. Por meio da figura podemos verificar que o uso de frameworks, entregas frequentes/ contınuas e backlog são as práticas mais utilizadas, contando com $78,6 \%$ indicações cada. As demais práticas, com o seu respectivas porcentagens de indicações, são: padrões de código (57,I\%), releases curtos $(64,3 \%)$, reuso de software $(42,9 \%)$, integração contínua $(28,6 \%)$, uso de indicadores de desempenho (42,9\%). e Uso de logs e estatísticas (35,7\%).

O uso de frameworks está entre as práticas mais utilizadas, uma vez que agiliza o processo de desenvolvimento ao retirar a necessidade de implementar algo que já está pronto, sendo uma das práticas mais importantes para o sucesso de uma startup (Giardino et al., 20I4). Outra prática popular é a realização de entregas frequentes/contínuas, pois permitem um ciclo de desenvolvimento mais rápido, alinhado ao processo empregado pelas metodologias ágeis, além de acelerarem o ciclo de feedback com o cliente, permitindo que ajustes sejam feitos de forma muito mais rápida para atender as demandas dos clientes. O uso de backlog também está entre as práticas mais utilizadas, sendo que esta é uma das principais práticas da metodologia Scrum, a metodologia mais utilizada entre os respondentes. 


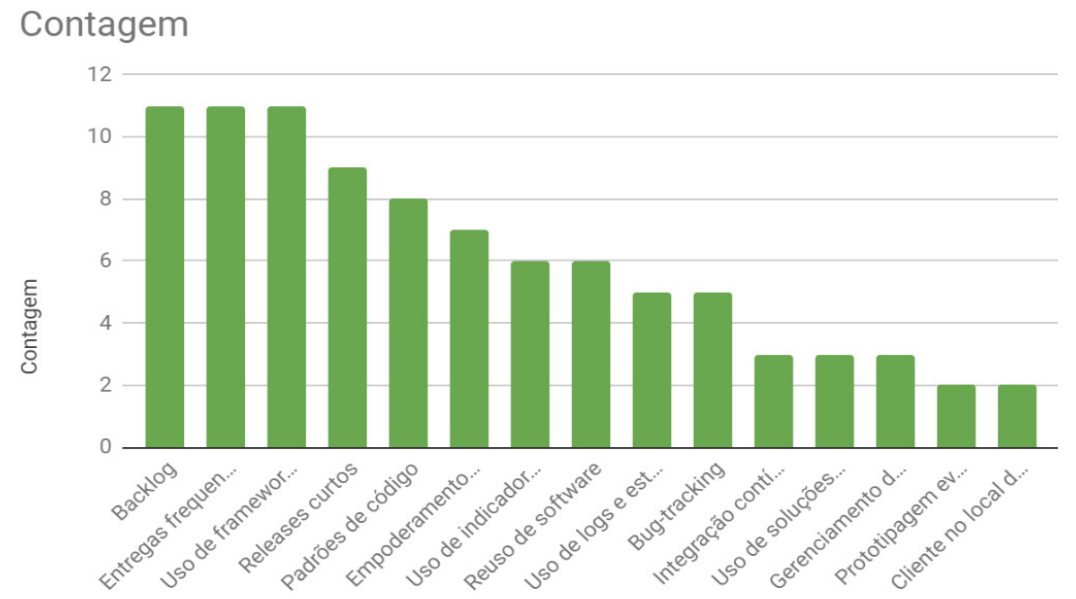

Gráfico 8 -Práticas de desenvolvimento utilizadas por startups

No Gráfico 9 são apresentadas as ferramentas de gerenciamento de projeto que as startups utilizam. Trello aparece como a mais popular, com 7 indicações, seguida por Atlassian Jira com 6, Asana com 4. As ferramentas TeamWork, Club House, Bitbucket pipelines e Sonarquibe não estavam listadas mas foram indicadas apenas I vez. Mingle, Pivotal Tracker, RedMine e Basecamp não foram indicadas por nenhuma das startups.

O motivo do Trello ser a ferramenta mais popular provavelmente se deve ao fato de o mesmo ser organizado da mesma forma em que o Kanban é executado, isto é, por meio de cartões de transitam entre painéis, além de ser uma ferramenta gratuita no plano mais básico. Essa similaridade facilita o seu uso, sendo a facilidade de uso o atributo que traz maior satisfação (Azizyan et al., 20II), e visto que a técnica Kanban é amplamente utilizada e está fortemente conectada a metodologia Scrum, que por sua vez é a metodologia mais usada.

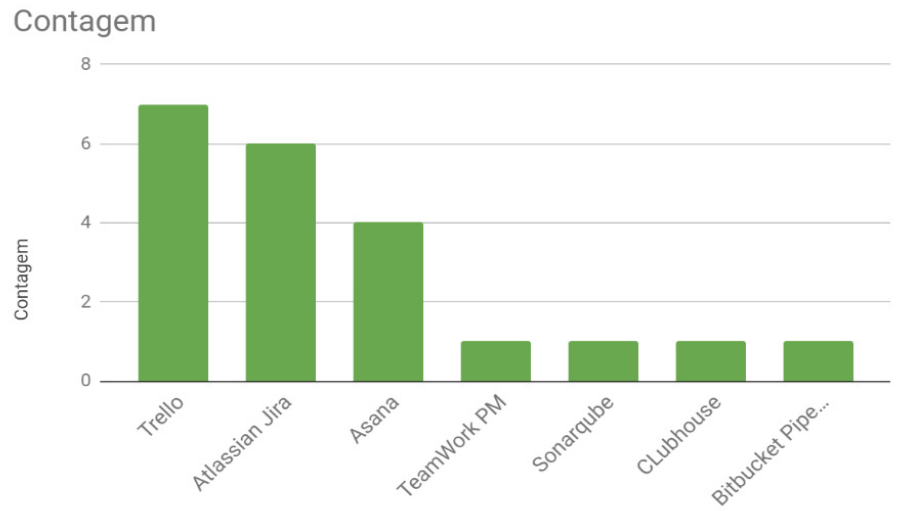

Gráfico 9 - Ferramentas de gerenciamento de projetos utilizadas por startups.

Também foi perguntado discursivamente as startups quais os principais pontos fortes da Gestão de Projetos em seu ambiente organizacional. Quatro das empresas entrevistadas apresentou respostas relacionadas a comunicação das equipes. Quatro empresas indicaram a utilização de metodologias ágeis. Individualmente foram apresentadas respostas relacionadas a bom gerenciamento do cronograma, entrega de valor para o cliente, entregas constantes e atividades automatizadas. Uma empresa não soube informar. 
Outra questão discursiva apresentada foi "Quais os principais pontos fracos da sua Gestão de Projetos? “. Duas empresas relataram problema com estabelecimento de prazos. Uma afirmou que por causa do time pequeno há uma sobrecarga de funções e nenhuma metodologia que experimentou aliviou a relação esforço x tempo. Outra empresa apontou a Qualificação dos recursos humanos e cultura da região como principal ponto fraco da sua startup. Além disso, outra organização relatou que na busca de novos projetos, desfoca do projeto corrente. Dificuldade em gerenciar todos os indicadores de desenvolvimento também foi apontado com um problema. Estrutura centralizadora, dificuldade em elaborar Roadmap foram indicados como pontos fracos. Uma empresa declarou que realização de testes automáticos e falta de equipe de teste.

A última questão discursiva foi "Dentro do processo como um todo, na sua visão, qual a maior dificuldade enfrentada pela equipe na condução do projeto? ". Três empresas apontaram mudança nos requisitos como maior dificuldade. Duas empresas afirmaram que sua maior dificuldade são a realização de testes. Também foram relatados Gerenciamento de riscos, Gerenciamento de stakeholders, Planejamento de curto prazo, Baixo conhecimento dos novos frameworks, exigências do mercado, adaptação a gerenciamento de projetos, e Código legado.

\section{Considerações finais}

A principal contribuição deste trabalho refere-se à condução do survey com startups pernambucanas que tenham entre suas atividades principais o desenvolvimento de software. Esta pesquisa empírica viabilizou caracterizar o estado do gerenciamento de projetos nestas organizações, a partir das metodologias, ferramentas, técnicas e práticas utilizadas. O estudo também permitiu caracterizar o perfil das pessoas envolvidas no cenário através do tempo de experiência que possuem com desenvolvimento de software, e qual o nível de escolaridade que possuem. Além do mais, a aplicação do survey possibilitou descrever o perfil das startups pernambucanas, dessa forma por intermédio do mesmo é possível identificar qual a média de colaboradores que estas startups possuem, em qual nível de maturidade se encontram, quais delas possuem algum tipo de investimento externo e quais fazem parte de algum grupo ou rede de colaboração.

Por fim, este trabalho possibilita proporcionar um grupo de evidências que foram obtidas por meio do survey para os pesquisadores e profissionais da indústria que buscam entender o contexto de gerenciamento de software em startups.

Baseado nisso, espera-se que seja provocado o interesse na realização de outros trabalhos que abordem o gerenciamento de projetos em startups.

A principal dificuldade encontrada durante a elaboração deste trabalho está relacionada principalmente àquelas atividades na qual foi necessário o envolvimento de terceiros. Durante a condução do survey, a principal dificuldade refere-se a baixa taxa de respostas, sendo este um problema conhecido na realização deste tipo de estudo. Para minimizar este efeito, o survey foi elaborado para que pudesse ser respondido rapidamente, sendo estimado o tempo médio para resposta do mesmo em Io minutos. Ainda assim, a taxa de resposta foi baixa, com apenas $9,42 \%$, mesmo após ter enviado o convite para participação no questionário mais de uma vez para todas as startups. 
Devido às limitações e lacunas encontradas durante a execução desta pesquisa, é viável destacar algumas oportunidades de trabalhos futuros. A partir da pesquisa exploratória é possível verificar que ainda existe uma lacuna em relação a quantidade de estudos empíricos a ser preenchida (Sutton, 2000). Assim sendo, é recomendado a execução de estudos que busquem criar cenários onde técnicas, práticas e ferramentas possam ser validadas e, essencialmente, comparadas.

Em razão da baixa taxa de resposta do survey, seria proveitoso realizar um novo estudo, talvez com um período de tempo maior, para identificar o perfil de mais startups pernambucanas. Outra possibilidade seria investigar as questões que foram realizadas, obtendo por exemplo, as motivações das escolhas de cada prática, técnica ou ferramenta.

\section{Referências}

ABSTARTUPS. Estatisticas. 20I9. Disponível em: <https://star-tupbase.abstartups.com.br/ stats $>$. Acesso em: Junho, 2019.

ASSOCIAÇÃO BRASILEIRA DE STARTUPS. Manual sobre conceitos, metodologias e investimentos em startups. Disponível em: . Acesso em: Setembro, 2019

ALVES, C.; PEREIRA, S.; CASTRO, J. A study in market-driven requirements engineering. In: WER, 2006. and needs. In: Agile Conference (AGILE), 20II, IEEE, 20II, p. 29-38.

AZIZYAN, G.; MAGARIAN, M. K.; KAJKO-MATSSON, M. Survey of agile tool usage and needs. In: Agile Conference (AGILE), 20II, IEEE, 20II, p. 29-38.

BLANK, Steve. The four steps to the epiphany. K\&S Ranch, 2013.

BUCHANAN, D. A.; BODDY, D. The expertise of the change agent: public performance and backstage activity. Prentice Hall London, 1992.

CARMEL, E. Time-to-completion in software package startups. In: 1994 Proceedings of the Twenty-Seventh Hawaii International Conference on System Science 498-507.

CAVAlCANTE, B. Diretrizes para o Desenvolvimento de Software em Startups. 20I8.219 f. Dissertação (Mestrado) - Curso de Ciência da Computação, Departamento de Informática,, Universidade Estadual de Maringá , Maringá, 2018.

COLLYER, S.; WARREN, C. M. Project management approaches for dynamic environments. International Journal of Project Management, v. 27, n. 4, p. 355-364, 2009.

CROWNE, M. Why software product startups fail and what to do about it. evolution of software product development in startup companies. In: Engineering Management Conference, 2002. IEMC'02. 2002 IEEE International, IEEE, 2002, p. 338-343.

DOM CABRAL (Nova Lima) (Org.). Causa da mortalidade das startups brasileiras: como 
aumentar as chances de sobrevivência no mercado. 20I5. Disponível em: $<$ https://www.fdc.org. br/conhecimento/blog>. Acesso em: 27 mar. 2019.

ETHIRAJ, S. K.; KALE, P.; KRISHNAN, M. S.; SINGH, J. V. Where do capabilities come from and how do they matter? A study in the software services industry. Strategic management journal, v. 26, n. I, p. 25-45, 2005.

FORZA, C. Survey research in operations management: a process-based perspective. International journal of operations \& production management, v. 22, n. 2, p. 152-194,2002.

FREDERIKSEN, L.; DAVIES, A. Vanguards and ventures: Projects as vehicles for corporate entrepreneurship. International Journal of Project Management, v. 26, n.5, p. 487-496, 2008.

GIL, ANTÔNIO CARLOS. Métodos e técnicas de pesquisa social. São Paulo: Atlas, I999.

GITAHY, Yuri. O que é uma startup?. Revista Exame, Out. 20Io. Disponível em: <http:/goo.gl/ zL4Mgj>. Acesso em Março, 20I9.

KERZNER, H. Gestão de projetos. Bookman, 2006.

KLEPPER, S. Entry, exit, growth, and innovation over the product life cycle. The American economic review, v. 86 , n. 3 , p. 562-583, 1996.

KLOTINS, E.; UNTERKALMSTEINER, M.; GORSCHEK， T. Software engineering knowledge areas in startup companies: a mapping study. In: International Conference of Software Business, Springer, 20I5, p. 245-257.

LARSON, E. W.; GRAY, C. F. Project management: The managerial process. 20II.

LIGA VENTURES (Brasil). Liga Insights Ecossistema Pernambuco Recife, fev. 2019.

NGUYEN-DUC, A.; Shah, S. M. A.; Ambrahamsson, P. Towards an early stage software startups evolution model. In: Software Engineering and Advanced Applications (SEAA), 2016 42th Euromicro Conference on, IEEE, 20I6, p. I20-I27.

OCH DAG, J. N. Elicitation and management of user requirements in market-driven software development. Dissertação de Mestrado, 2002.

PATERNOSTER, N.; GIARDINO, C.; UNTERKALMSTEINER, M.; GORSCHEK, T.; ABRAHAMSSON, P. Software development in startup companies: A systematic mapping study. Information and Software Technology, v. 56, n. IO, p. I200-I2I8, 2014.

PROJECT MANAGEMENT INSTITUTE - PMI. Project Management Body of

Knowledge (PMBOK) 5. ed. Newtown Square: Project Management Institute, 2012 
PMI. Project Management Institute. v. 20I6. n. I5 jan.20I6. p. O que é o PMI?

SANTOS, M. C. F. R. D. O ecossistema de startups de software da cidade de São Paulo. Tese de Doutoramento, Universidade de São Paulo, 2016.

SHENHAR, A. J.; DVIR, D.; LEVY, O.; MALTZ, A. C. Project Success: A Multidimensional Strategic Concept. Long Range Planning, v. 34, n. 6, p. 699-725, 2002.

SUTTON, STANLEY M.. 2000. The Role of Process in a Software Start-up. IEEE Software. I7, 4 (Jul. 2000), 33-39.

VON HIPPEL, E. Successful and failing internal corporate ventures: An empirical analysis. Industrial Marketing Management, v. 6, n. 3, p. I63-I74, 1977.

WILLIAMS, T. Assessing and moving on from the dominant project management discourse in the light of project overruns. Engineering Management, IEEE Transactions on, v. 52, n. 4, p. 497-508, 2005. 